\title{
Natural Convection Study by the Direct Integration of the Momentum and Energy Equations
}

\author{
Mohamed Bachiri ${ }^{* 1}$ and Ahcene Bouabdallah ${ }^{2}$ \\ *1 Technical Sciences Department, Faculty of Technology, University of Amar Telidji \\ B.P 37 G, Laghouat 03000, Algeria \\ ${ }^{2}$ Thermodynamics and Energetical Systems Laboratory, Faculty of Physics, USTHB \\ B.P 32 El Alia, 16111 Bab Ezzouar-Algiers, Algeria \\ Email: mabach73@yahoo.fr
}

\begin{abstract}
In this paper, we investigate theoretically the natural thermoconvection phenomena near a vertical plate for two thermal boundary conditions at the sidewalls. A specifically developed analytical model based on the new integral method form is used for the solution of the momentum and energy transfer governing equations. From an adequate choice of velocity and temperature expressions, two new Nusselt-Rayleigh relations, depending on the sidewalls conditions, are derived for all Prandtl numbers. Comparisons among the different thermal configurations considered are reported. In particular, with reference to the typical configuration wherein the sidewalls are isothermal and in uniform heat flux, it is found that the NusseltRayleigh coefficient given via this method indicates a good agreement with the theoretical and experimental results for all Prandtl numbers.
\end{abstract}

Keywords: Natural thermoconvection, Vertical plate, Integral method, Isothermal, Uniform heat flux.

\section{INTRODUCTION}

The investigation of the natural convection flows of an incompressible fluid near a surface has many important applications in engineering, such as aerodynamics, the boundary layer along a liquid film condensation process and the cooling process of the electronic components. In particular, the natural convection along a semi-infinite vertical wall has been studied extensively in the existing literature, including: Squire [1] who has used the integral method and obtained the Nusselt number expression, in case of an isothermal vertical plate. Ostrach [2] studied numerically the natural convection and calculated the Nusselt number for various Prandtl numbers, Ede [3] analyzed the well-known experimental data in the special range of Rayleigh number and showed, that they are rather well described by the generalized formula for the local Nusselt number. Based on the analysis of various experimental data, Churchill and Usagi [4] gave an accurate correlation relating Nusselt number to Prandtl and Rayleigh numbers. For vertical plates in a turbulent regime with a constant wall temperature, Cebeci and Bradshaw [5] proposed a Nusselt expression by solving the integral equations of motion and energy. Bejan [6] investigated the steady state flow behavior using a scaling analysis to obtain scaling laws for the dominant parameters characterizing this kind of flow and it was found that these scaling laws are in a good agreement with the numerical and theoretical results. Proceeding from the integral equations and using the dimensional method, the analysis of different regimes of free-convective flow near an isothermal vertical plate due to the simultaneous action of temperature and concentration gradients was carried out in [7]. Based on the similarity theory, Sparrow and Gragg [8] carried out investigations in the wide range of Prandtl values and suggested the limiting dependencies for the local Nusselt number. Using the integral method, Sparrow [9] obtained a formulation of the local Nusselt number by making the same assumptions concerning the scales of the boundary layer as in [1]. Churchill and Ozoe [10, 11] generalized the data of works $[4,8,13]$ and suggested an accurate Nusselt number formula which is valid for all Prandtl number in a laminar flow. Fujii [14] analyzed the solutions obtained by many researchers [8, 15, 16-19] and proposed a theoretical Nusselt number correlation, in case of a constant heat flux. Recently, Oleg, Martynenko and Khramtsov [20] studied natural convection problems for different geometries by means of analytical, numerical and experimental analysis.

It is known that the integral method may be used to solve analytically the equations governing the thermoconvection process with a relatively good efficiency. From this method, we try to determine, via ad hoc formulae of the velocity and temperature profiles, the Nusselt-Rayleigh coefficients for all Prandtl numbers when the sidewalls are isothermal and uniform heat flux. From this, we use the same velocity profile form adopted by the similarity method in our study [21]. The 
objective is to check the validity of the behavior of the flow and to determine the heat transfer rate, in order to compare the conduction regime versus the convective regime by evaluating the Nusselt number.

\section{BASIC FLOW AND FORMULATIONS}

We consider natural convection evolving along a vertical plate, where the coordinate $y$ is taken positive upward in the vertical direction, and the direction $x$ is taken normal to the surface of the plate. The plate is placed in an extensive and quiescent fluid, and is maintained under an isothermal wall or a uniform heat flux on a wall condition, See Figure 1. We are interested in the study of a steady state laminar natural convection of an incompressible fluid with constant proprieties. Within the framework of the Boussinesq approximations, the equations of motion for the velocity $V(u$, $v$ ) and the temperature $T$ take the form

$$
\frac{\partial u}{\partial x}+\frac{\partial v}{\partial y}=0
$$

$u \frac{\partial v}{\partial x}+v \frac{\partial v}{\partial y}=v \frac{\partial^{2} v}{\partial x^{2}}+g \beta\left(T-T_{\infty}\right)$

$u \frac{\partial T}{\partial x}+v \frac{\partial T}{\partial y}=\alpha \nabla^{2} T$

For complete description of the vertical natural-convective boundary layer, Eqs. (1), (2), and (3) should be supplemented with the boundary conditions:

a- constant wall temperature

$u(y, 0)=v(y, 0)=v(y, x \rightarrow \infty)=0$,

$T(y, 0)=T_{0}, T(y, x \rightarrow \infty)=T_{\infty}$.

b- constant heat flux density on a wall

$u(y, 0)=v(y, 0)=v(y, x \rightarrow \infty)=0$,

$-\alpha \frac{d T}{d x}(y, 0)=q^{\prime \prime}, T(y, x \rightarrow \infty)=T_{\infty}$.

where $v$ is the kinematic viscosity, $\alpha$ is the thermal diffusivity, $\beta$ is the volumetric thermal expansion coefficient, $g$ is the gravitational acceleration, $T_{\infty}$ is the uniform temperature far away from the plate, and $q^{\prime \prime}$ is the heat flux density on a wall. In opposition to the similarity method which seeks to find a local solution of the boundary layer differential equations, the integral method proceeds with direct integration of the velocity and temperature profiles within the boundary layer.

In the context of our work, it is a global method based on the analytical integration of the momentum and energy boundaries layer differential equations. The problem may be expressed as
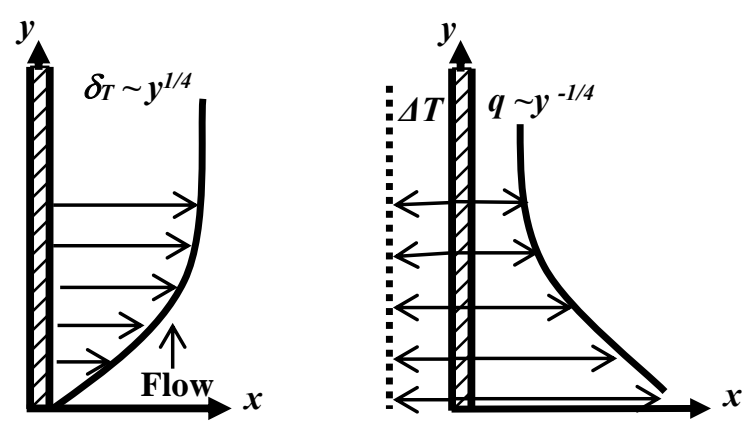

(a)
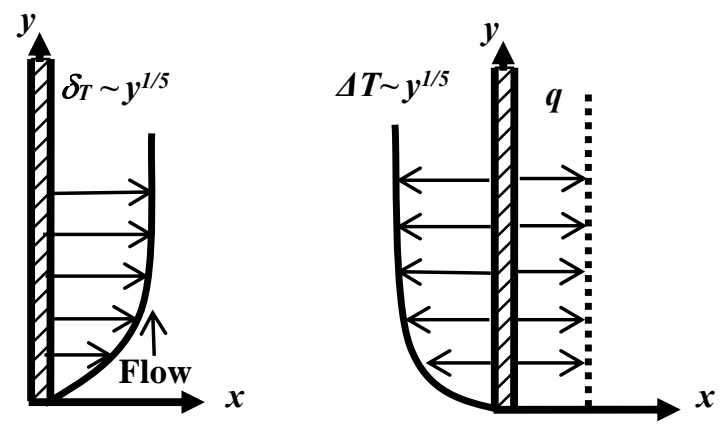

(b)

Figure 1. Structure of a natural convection flow and a thermal boundary layer on a vertical surface; (a) for an isothermal wall and (b) for a uniform heat flux on a wall

$\frac{1}{2} \frac{d}{d y} \int_{0}^{\infty} v^{2} d x=-v\left(\frac{\partial v}{\partial x}\right)_{x=0}+g \beta \int_{0}^{\infty}\left(T-T_{\infty}\right) d x$

$\int_{0}^{\infty} v \frac{d}{d y}\left(T_{\infty}-T\right) d x=\alpha\left(\frac{\partial T}{\partial x}\right)_{x=0}$

Bejan [6] gives a detailed study of the scales intervening in the momentum and energy equations, combined with a qualitative analysis taking account of the fluid properties influence on the forces balance appearing in the momentum equation. This reasoning mode, apparently rarely quoted in current research of natural convection, proposes new formulations completely different from that found by many researchers since 1930.

From the Bejan's scale analysis, it is found that the thermoconvective boundary layer geometry for fluids with $\operatorname{Pr}>1$ differs completely from that of fluids with $\operatorname{Pr}<1$. Therefore, it is clear that two size scales must obligatorily control the velocity profile form: one $(\operatorname{Pr}<1)$ by the shearing layer thicknesses of the wall $\delta_{v}$ and the thermal layer thickness $\delta_{T}$, the other $(\operatorname{Pr}>1)$ by the dynamic boundary layer thickness $\delta$ and thermal layer thickness $\delta_{T}$.

In order to avoid any kind of ambiguity on the difficulties of connecting the velocity profile form depending on two thicknesses at the same time, we refer qualitatively to Bejan's scale analysis of the velocity behavior in the thermoconvective boundary layer. It is clear that in case of $\mathrm{Pr}$ $\sim 1$ where the dynamic and thermal boundary layer thicknesses are almost equal $\left(\delta \sim \delta_{T}\right)$, the velocity formula can be written only according to one of the thicknesses. 
Indeed, the velocity and temperature profiles obey to this case are given by the following expressions:

$$
\begin{aligned}
& v=V\left(\frac{x}{\delta}\right)\left(\exp \left(-\left(\frac{x}{\delta}\right)^{2}\right)+\frac{1}{2} \exp \left(-\frac{5}{2} \frac{x}{\delta}\right)\right) \\
& T-T_{\infty}=\Delta \operatorname{Terfc}\left(-\frac{1}{2} \frac{x}{\delta_{T}}\right)
\end{aligned}
$$

From Bejan's scale analysis, we found that $V \sim y^{1 / 2}$ and $\delta_{T} \sim \delta \sim y^{1 / 4}$ which indicates the scales of the vertical velocity and the thermal and dynamic boundary layer thicknesses, respectively.

\section{RESULTS AND DISCUSSION}

In the relevant situations involving natural convection acting by free cooling on a vertical plate, we consider two important cases: uniform wall temperature, and uniform wall heat flux.

\subsection{Uniform wall temperature}

Replacing the profiles of Eqs.(6) and (7) in the integral equations system of Eqs. (4) and (5), we arrive at the following results:

$\left\{\begin{array}{l}g \beta \Delta T=0.115+1.329 v \\ \alpha=0.0886\end{array}\right.$

The local Nusselt number is given by the following expression

$$
N u_{y}=\frac{q^{\prime \prime}}{T_{0}-T_{\infty}} \frac{y}{k}=\left[\frac{\alpha \nu}{g \beta \Delta T}\right]^{1 / 4} R a_{y}{ }^{1 / 4}
$$

where $q^{\prime \prime}$ is the conductive heat flux, $k$ is the thermal conductivity coefficient, $T_{0}$ is the wall temperature, and $R a_{y}=\frac{g \beta \Delta T y^{3}}{\alpha v}$ is the Rayleigh number.

From the Eqs. (8), (9) and (10) relations, we obtain the local Nusselt number according to the influence parameters such as $R a_{y}$ and $\mathrm{Pr}$

$$
N u_{y}=0.508\left[\frac{\operatorname{Pr}}{0.976+\operatorname{Pr}}\right]^{1 / 4} R a_{y}^{1 / 4}
$$

Taking account of the asymptotic limits related to $\mathrm{Pr}$, the global Nusselt number $N u_{H}$ corresponding to two interesting physical situations is given as

$$
\begin{array}{ll}
-\operatorname{Pr} \rightarrow 0 & N u_{H}=0.68\left(\operatorname{Pr} R a_{H}\right)^{1 / 4} \\
-\operatorname{Pr} \rightarrow \infty & N u_{H}=0.677 R a_{H}{ }^{1 / 4}
\end{array}
$$

We note that these limiting expressions are anticipated correctly by Bejan's qualitative study, [6].

Churchill and Chu [11], on the basis of analysis of a large number of works, suggested the following correlations

$$
N u_{y}=0.503\left[1+\left(\frac{0.492}{\operatorname{Pr}}\right)^{9 / 16}\right]^{-4 / 9} R a_{y}{ }^{1 / 4}
$$

The integral method developed by Karman applied by Squire [1] in the problem of the natural convection obtained the following dependence for the local Nusselt number:

$N u_{y}=0.508\left[\frac{\operatorname{Pr}}{0.952+\operatorname{Pr}}\right]^{1 / 4} R a_{y}{ }^{1 / 4}$

From the numerical solution of the basic differential equations, Le Fevre [12] also suggested a general form of empirical dependence for the local Nusselt number:

$$
N u_{y}=\frac{3}{4}\left[\frac{\operatorname{Pr}}{2.434+4.884 \operatorname{Pr}^{1 / 2}+4.952 \operatorname{Pr}}\right]^{1 / 4} R a_{y}{ }^{1 / 4}
$$

It should be noted that many researchers have used the integral method approximation presented previously, with different choices of the velocity and temperature profiles, to obtain the heat transfer coefficient law. Figure 2 gives a comparison of the Nusselt number theoretical expressions, established by integral method, with the Churchill-Chu's experimental correlation for a wide range of $\mathrm{Pr}$ values and in case of the laminar natural convection of an isothermal

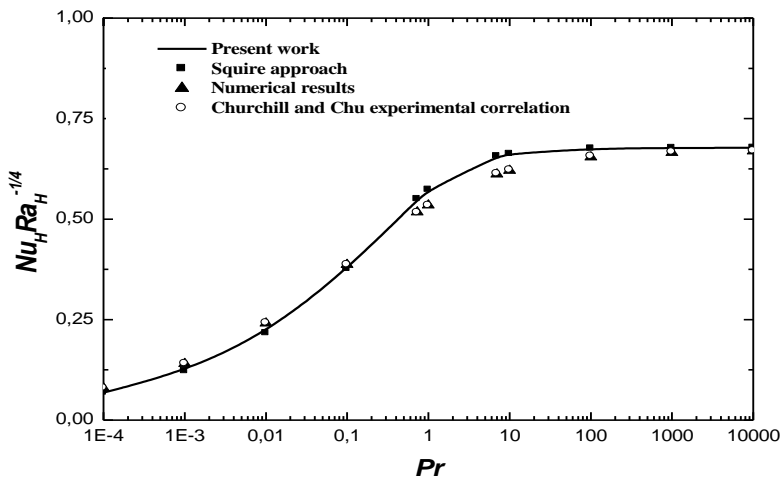

Figure 2. Average Nusselt number relating to uniform isothermal vertical wall in laminar natural convection

On this figure, the results of the present work are shown to match closely with results obtained by Squire [1], and the experimental data. Bejan's scale analysis demonstrates geometrically and phenomenologically that the velocity profile depends not only on the dynamic thickness which is the case of the profiles chosen by Squire, and the present study, but also on the two thicknesses $\delta_{v}(\operatorname{Pr}<1)$ and $\delta(\operatorname{Pr}>1)$. We explain this discordance by the fact that the choice of velocity and temperature profiles always remains approximate and based on a simple theoretical analysis. 


\subsection{Uniform wall heat flux}

Using the integral method equation and keeping the same velocity and temperature profiles, we find

$\left\{\begin{array}{l}g \beta \frac{q^{\prime \prime}}{k}=0.129+1.329 v \\ \alpha=0.129\end{array}\right.$

where $R a^{*}{ }_{H}=\frac{g \beta q^{\prime \prime} H^{4}}{\alpha v k}$, and $q^{\prime \prime}=$ constant.

From these last equations, we derive the local Nusselt number as

$N u_{y}=0.627\left[\frac{\operatorname{Pr}}{\operatorname{Pr}+0.749}\right]^{1 / 5} R a_{y}^{* 1 / 5}$

Taking account of $N u_{H}$ corresponding to two interesting physical situations verifies Bejan's scale laws.

$$
\begin{array}{ll}
-\operatorname{Pr} \rightarrow 0 & N u_{H}=0.83\left(\operatorname{Pr} R a_{H}^{*}\right)^{1 / 5} \\
-P r \rightarrow \infty & N u_{H}=0.78 R a_{H}^{*}{ }_{H}^{1 / 5}
\end{array}
$$

Churchill and Ozoe [10] generalized the experimental results by many researchers and proposed the following formula which is valid for all $\operatorname{Pr}$ number in a laminar flow:

$$
N u_{y}=0.563\left[1+\left(\frac{0.437}{\operatorname{Pr}}\right)^{9 / 16}\right]^{-4 / 9} R a_{y}^{*}{ }^{1 / 5}
$$

T. Fujii and M. Fujii [14] analyzed the solutions obtained by various researchers [8, 15, 16-19] and suggested the following expression of Nusselt number:

$N u_{y}=\left[\frac{1}{4+9 \operatorname{Pr}^{1 / 2}+10 \operatorname{Pr}}\right]^{1 / 5} R a_{y}^{*}{ }^{1 / 5}$

The application of the direct integration method in case of a constant heat flux on a surface also allowed us to find the temperature distribution corresponding to the given value of the heat flux.

$$
\frac{\left(T_{0}(y)-T_{\infty}\right)}{q^{\prime \prime} / k}=1.595\left[\frac{\operatorname{Pr}}{\operatorname{Pr}+0.749}\right]^{-1 / 5} y R a_{y}^{*-1 / 5}
$$

Figure 3 shows that the temperature on the wall at $y=0$ increase when $\operatorname{Pr}>1$, and remains almost the same when $\operatorname{Pr}<1$. Also, along the wall, we see the same temperature distributions curves for all fluids of $\operatorname{Pr}<1$, but for fluids of $\operatorname{Pr}>1$ the curves increase but the temperature distributions decrease approximately by the same variations. Figure 4 represents the variation of the heat transfer coefficient depending on $\operatorname{Pr}$ and $R a$. The results obtained by the analytical solution procedure introduced in the preceding section are consistent with that in the literature. The graphical output of the results reveals that there is a good agreement of the average Nusselt number expressions with that obtained by Fujii's theoretical correlation and Churchill-Ozoe's experimental correlation.

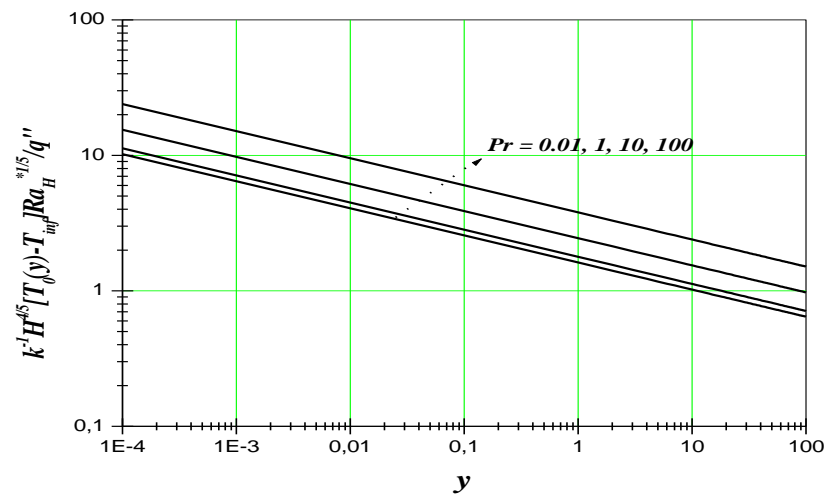

Figure 3. Temperature variation corresponding to the given values of the heat flux

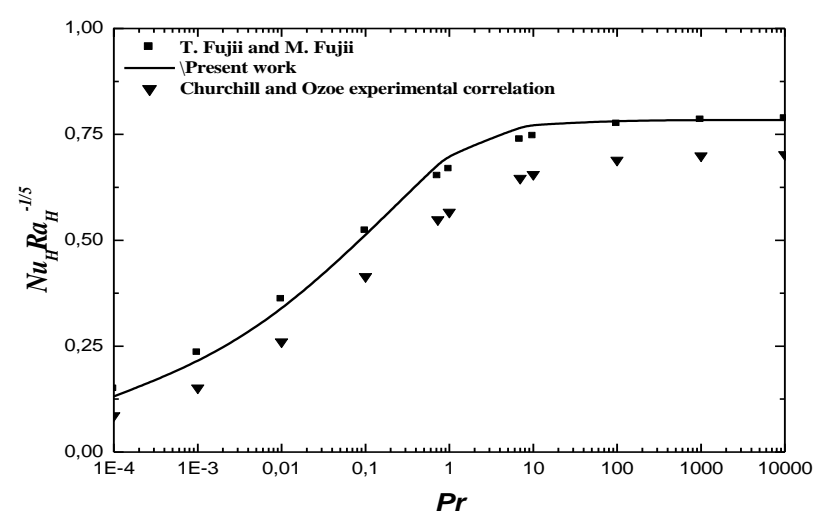

Figure 4. Average Nusselt number relating to uniform heat flux of vertical wall in laminar natural convection

\section{CONCLUSION}

The study of the thermoconvection, particularly the steadystate natural convection flow over a semi-infinite vertical plate maintained at a uniform temperature and uniform heat flux are carried out using the new direct integration of momentum and energy equations. This method is different from those used by many researchers. Our approach proceeds from phenomenological considerations based on Bejan's work. From the behaviour of the phenomena, analytical formulae of the velocity and temperature profiles are proposed in case of $\mathrm{Pr} \sim 1$. This condition permits us to solve the integral equations system in order to generalize the heat transfer coefficients expressions for all $\mathrm{Pr}$ values in the two relevant physical situations; isothermal vertical wall and uniform heat flux wall.

These results appear very satisfactory when we compare them with the theoretical and experiment correlations.

Consequently, we can conclude that the analytical form of the selected velocity profile, used by both similarity and integral approaches, meets the phenomenological requirements 
imposed by the natural convection mechanism acting on a vertical wall in steady-state laminar regime.

\section{REFERENCES}

[1] H. B. Squire, "Integral solution," in Modern Developments in Fluid Dynamics, vol. 2, S. Goldstein ed., Dover, New York, USA: Academic press, pp. 641-643, 1965.

[2] S. Ostrach, "An analysis of laminar free convection flow and heat transfer about a flat plate parallel to the direction of the generating body force," Natio. adv. Commit. for Aeronautics, Technical note 2635, Feb. 1952.

Available: http://naca.central.cranfield.ac.uk/reports/1952/nacatn-2635.pdf.

[3] A. J. Ede, "Advances in free convection," Advances in Heat Transfer, vol. 4, no. 1, pp. 1-64, 1967. DOI: 10.1016/S0065-2717(08)70272-7.

[4] S. W. Churchill and R. Usagi, "A general expression for the correlation of rates of transfer and other phenomena," AIChE Journal, vol. 18, no. 6, pp. 11211128, Nov. 1972. DOI: 10.1002/aic.690180606.

[5] T. Cebeci and P. Bradshaw, "Buoyant flows," in Physical and computational aspects of convective heat transfer, Springer Study edition, New York, USA: Springer, 1988, pp. 263-300. DOI: 10.1007/978-14612-3918-5 9 .

[6] A. Bejan, "External natural convection," in Convection Heat Transfer, $\mathbf{3}^{\text {rd }}$ ed., New York, USA: Wiley, John \& Sons, Inc., 2004, pp. 156-218.

[7] A. Mongruel, M. Cloitre, and C. Allain, "Scaling of boundary layer flows driven by double-diffusive convection," Int. J. Heat Mass Transfer, vol. 39, no. 18, pp. 3899-3910, Dec. 1996. DOI: 10.1016/00179310(96)00054-3.

[8] E. M. Sparrow and J. L. Gregg, "Buoyancy effects in forced-convection flow and heat transfer," J. Appl. Mech., vol. 26, pp. 133-134, 1959.

[9] E. M. Sparrow, "Laminar free convection on a vertical plate with prescribed nonuniform wall heat flux or prescribed nonuniform wall temperature," Natio. adv. Commit. for Aeronautics, Technical note 3508, 1955.

[10] S. W. Churchill and H. Ozoe, "A Correlation for laminar free convection from a vertical plate," J. Heat Transfer, Trans. ASME, vol. 95, no. 4, pp. 540-541, Nov. 1973. DOI: $10.1115 / 1.3450105$.

[11] S. W. Churchill and H. H. S. Chu, "Correlating equations for laminar and turbulent free convection from a vertical plate," Int. J. Heat Mass Transfer, vol. 18 , no. 11, pp. 1323-1329, Nov. 1975. DOI: 10.1016/0017-9310(75)90243-4.

[12] E. J. Le fevre. "Laminar free convection from a vertical plane surface," Presented at the 9th International Congress on Applied Mechanics, Brussels, 1956, pp. 1-168.

[13] J. R. Selman and J. Newman, "High Sc limit of free convection at a vertical plate with uniform flux condition," J. Heat Transfer, vol. 93, no. 4, pp. 465466, Nov. 1971. DOI: $10.1115 / 1.3449848$.

[14] T. Fujii and M. Fujii, "The dependence of local Nusselt number on Prandtl number in the case of free convection along a vertical surface with uniform heat flux," Int. J. Heat Mass Transfer, vol. 19, no. 1, pp.

121-122, Jan. 1976. DOI: $\underline{10.1016 / 0017-}$ 9310(76)90020-X.

[15] K. S. Chang, R. G. Akins, L. Burris and S. G. Bankoff, "Free convection of a low Prandtd number fluid in contact with uniformly heated verticale plate," Argonne National Lab., Rep. ANL-6835, Jan. 1964. DOI: $10.2172 / 4031447$.

[16] B. Gebhart, "Effects of viscous dissipation in natural convection," J. Fluid Mech., vol. 14, no. 2, pp. 225232, Oct. 1962. DOI: 10.1017/S0022112062001196.

[17] T. Fujii and H. Uehara, "Laminar natural convective heat transfer from the outer surface of a vertical cylinder," Int. J. Heat Mass Tran., vol. 13, no. 3, pp. 607-615, Mar. 1970. DOI: $10.1016 / 0017-$ 9310(70)90155-9

[18] H. K. Kuiken, "An asymptotic solution for large Prandtl number free convection," J. Eng. Math., vol. 2, no. 4 pp. 355-371, Oct. 1968. DOI: 10.1007/BF01579575.

[19] H. K. Kuiken, "Free convection at low Prandtl numbers," J. Fluid Mech., vol. 37, no. 4, pp. 785-798, July 1969. DOI: $10.1017 /$ S0022112069000887.

[20] Oleg G. Martynenko and Pavel P. Khramtsov, "Free convection on a plane," in Free-convective Heat Transfer, First ed., Berlin, Germany: Springe-Verlag, 2005, pp. 81-218. DOI: 10.1007/3-540-28498-2.

[21] M. Bachiri and A. Bouabdallah, "an Analytic investigation of the steady-state natural convection boundary layer flow on a vertical plate for a wide range of Prandtl numbers," Int. J. Heat Tran. Eng., vol. 31, no. 7, pp.608-616, June 2010. DOI: $\underline{10.1080 / 01457630903425908 .}$.

\section{NOMENCLATURE}

$g$

$H$

$k$

$N u_{H}$

$N u_{y}$

$\operatorname{Pr}$

$q^{\prime \prime}$

$R a_{H}$

$R a_{y}$

$T$

$T_{0}$

$T_{\infty}$

$u, v$

V

$x, y$

\section{Greek symbols}

$\alpha$

$\beta$

$\delta$

$\delta_{T}$

$\delta_{v}$

$v$ gravitational acceleration, m.s-2

reference length, $\mathrm{m}$

thermal conductivity, W.m-1. K-1

global Nusselt number

local Nusselt number

Prandtl number

heat flux density, W.m-2

global Rayleigh number

local Rayleigh number

temperature, $\mathrm{K}$

wall temperature, $\mathrm{K}$

ambient temperature, $\mathrm{K}$

velocities componets, m.s-1

velocity, m.s-1

coordinates, $\mathrm{m}$

thermal diffusivity, m2. s-1

thermal expansion coefficient, K-1

dynamic layer thickness, $\mathrm{m}$

thermal layer thickness, $m$

shearing layer thickness of the wall, $m$

kinematic vescosity, m2.s-1 